\title{
Exploring factors influencing occupational therapists' perception of patients' rehabilitation potential after acquired brain injury
}

\author{
Priscilla Lam Wai Shun, OT, MSc, PhD candidate ${ }^{1,2}$, Carolina Bottari, OT, $\mathrm{PhD}^{1,2}$, \\ Tatiana Ogourtsova, OT, MSc, $\mathrm{PhD}$ candidate ${ }^{2,3}$, Bonnie Swaine, $\mathrm{PT}, \mathrm{PhD}{ }^{1,2}$
}

1. Occupational Therapy, School of Rehabilitation, Faculty of Medicine, Université de Montréal, Québec, Canada

2. Centre for Interdisciplinary Research in Rehabilitation of Greater Montreal, Montréal, Québec, Canada

3. School of Physical and Occupational Therapy, Faculty of Medicine, McGill University, Montréal, Québec, Canada

This is the peer reviewed version of the following article: Lam Wai Shun, P., Bottari, C., Ogourtsova, T., Swaine, B. (2017) Exploring occupational therapists' perspective of factors influencing their assessment of rehabilitation potential after acquired brain injury. Australian Occupational Therapy Journal, 64(2), 149-158, DOI: 10.1111/1440-1630.12327, which has been published in final form at https://doi.org/10.1111/1440-1630.12327. This article may be used for non-commercial purposes in accordance with Wiley Terms and Conditions for Use of Self-Archived Versions. This article may not be enhanced, enriched or otherwise transformed into a derivative work, without express permission from Wiley or by statutory rights under applicable legislation. Copyright notices must not be removed, obscured or modified. The article must be linked to Wiley's version of record on Wiley Online Library and any embedding, framing or otherwise making available the article or pages thereof by third parties from platforms, services and websites other than Wiley Online Library must be prohibited. 


\section{Corresponding author:}

Priscilla Lam Wai Shun, OT, MSc, PhD candidate

Centre for Interdisciplinary Research in Rehabilitation of Greater Montreal

Lucie-Bruneau Rehabilitation Centre

2275, Laurier Avenue East, room 106.40

Montréal (Québec)

Canada H2H 2N8

Email: priscilla.lam.wai.shun@umontreal

\section{Conflict of interest statement}

The authors report no conflict of interest 


\section{Exploring factors influencing occupational therapists' perception of patients' rehabilitation potential after acquired brain injury}

\section{INTRODUCTION}

Stroke and traumatic brain injury are the most common acquired brain injuries (ABI) and leading causes of disability among adults (World Health Organization, 2003). Compelling evidence indicates that comprehensive multidisciplinary inpatient rehabilitation leads to an increased number of these persons achieving and maintaining optimal functioning within their environments (Cullen, Meyer, Aubut, Bayley, \& Teasell, 2014; Lindsay et al., 2010). However, there are worldwide reports indicating that inpatient rehabilitation is a limited resource that is not provided to all (Jourdan et al., 2013; Kennedy, Brock, Lunt, \& Black, 2012; Pereira et al., 2012). This leads to the challenging task of selecting appropriate patients for rehabilitation.

Variations within and across nations and regions in regard to the practice of patient referral and admission to inpatient rehabilitation after acute ABI have been observed (Ilett, Brock, Graven, \& Cotton, 2010; Swaine et al., 2011). In some service delivery models, such as in some parts of Australia, an inpatient rehabilitation assessor (usually a physician) determines acute care patients' eligibility for inpatient rehabilitation (Conroy, DeJong, \& Horn, 2009; Hayward, Aitken, Barker, \& Brauer, 2014; Kennedy et al., 2012). This assessor may or may not have input from acute care rehabilitation professionals. In other instances, such as in some parts of Canada, triage for rehabilitation is performed by acute care teams and rehabilitation professionals are involved at varying degrees in the referral process to inpatient rehabilitation (Cullen, 2007). Yet, in other cases, the decision to 
transfer a patient to inpatient rehabilitation may be dictated by health insurances and insurers may or may not consider physicians' or rehabilitation professionals' recommendations (Putnam et al., 2007).

Occupational therapists' main role in acute care settings has been viewed as facilitating safe and timely home discharge and referral to rehabilitation when discharge home is not possible (Britton, Rosenwax, \& McNamara, 2015; Crennan \& MacRae, 2010). In Montreal, Canada, occupational therapists working in acute care are routinely asked to perform early assessment of patients' rehabilitation potential (RP) and to participate in the referral process to rehabilitation. Rehabilitation professionals' involvement in early screening of ABI patients' rehabilitation needs has been advocated in clinical practice guidelines worldwide (ABIKUS Guideline Development Group, 2007; Casaubon et al., 2016; Government of South Australia, 2012; National Stroke Foundation, 2015; Schwamm et al., 2005). However, to our knowledge, no studies have yet examined occupational therapists' role in the process of early assessment of RP. Improving our understanding of occupational therapists' practices in this field may prove to be useful for academic and clinical education purposes, to foster reflection on practice for clinicians already involved in the assessment of RP, and for clinicians advocating for increased involvement of occupational therapists in this type of assessment.

This study is the first of a series of studies investigating the role of acute care occupational therapists in the assessment of RP. The objectives of this exploratory study were (1) to document the most important patient-related factors occupational therapists perceive as influencing ABI patients' RP for inpatient rehabilitation and (2) to explore whether additional factors, apart from patient-related factors, shape their perception of 
patients' RP.

\section{METHODS}

\section{Design}

A qualitative study with an interpretive description approach was used. Interpretive description is concerned with capturing themes within subjective experiential knowledge that lead to a description capable of informing discipline-specific clinical understanding of a phenomena of interest (Thorne, 2008). Studies based on interpretive description are grounded in clinical issues and recognise that the researcher's theoretical knowledge and clinical background will shape the inquiry as well as the analytic process. This study design was felt to be best suited to explore the subjective perspective of occupational therapists involved in the clinical assessment of RP and to elicit clinical knowledge on factors influencing their perception of patients' RP.

\section{Participants}

Purposive sampling was used to identify participants with insight into the phenomena of interest (Thorne, 2008). Key informants for this study were selected from the pool of occupational therapists working in ABI programs in three acute care and three inpatient rehabilitation hospitals in Montreal, the second most populous city in Canada. Acute care occupational therapists participate in the assessment of patients' RP early in the recovery process. However, because inpatient rehabilitation in Montreal is provided in freestanding hospitals, acute care occupational therapists never receive feedback on patients' outcome once patients are transferred to inpatient rehabilitation. Occupational therapists working in 
inpatient rehabilitation, on the other hand, observe how patients with varying levels of RP recover in inpatient rehabilitation. We felt the shared perspective of therapists from both settings would provide invaluable information regarding common factors influencing occupational therapists' perception of ABI patients' RP. Finally, participants with at least three years of experience with the ABI clientele were included in this study. All participants provided written informed consent. Ethical approval was obtained from the Research Ethics Boards of the McGill University Health Centre, Centre for Interdisciplinary Research in Rehabilitation of Greater Montreal, "Centre Hospitalier de l'Université de Montréal", "Hôpital du Sacré-Coeur de Montréal" and the "Centre de santé et de services sociaux Cavendish".

\section{Data collection}

A focus group was conducted using a consensus seeking technique called TRIAGE, i.e.

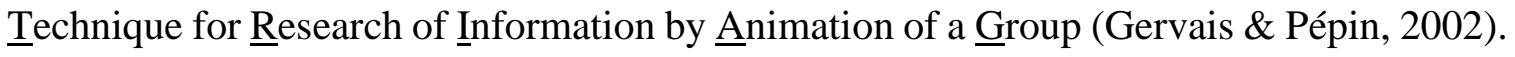
This technique draws upon the constructivist paradigm of social research and consists of an interactive technique used to obtain expert consensus. TRIAGE is increasingly used in rehabilitation research to gather expert opinion on relevant rehabilitation outcomes or on key dimensions of rehabilitation services in quality improvement efforts (Lamontagne, Routhier, \& Auger, 2013; Swaine, Dutil, Demers, \& Gervais, 2003; Veillette, Demers, Dutil, \& McCusker, 2009). TRIAGE is organised in two phases, an individual phase based on each participant's individual contribution and a group phase aimed at obtaining consensus. 


\section{Individual phase}

The individual phase incites participants to reflect on the phenomenon of interest and provides data for the subsequent group phase. For this study, each participant was asked to provide written responses to questions related to socio-demographic characteristics and to their clinical practice. Findings from this section were used for sample description. Participants were then asked to put into words their understanding of RP. Participants were also asked to respond to questions pertaining to three case scenarios developed for the purpose of this study: one of a patient with a traumatic brain injury, another of a patient with a brain tumour, and a third one of a patient with a stroke. The case scenarios were based on the authors' clinical experience and on the literature on factors known to be predictive of outcome. The scenarios described typical patients encountered by occupational therapists in $\mathrm{ABI}$ acute care and rehabilitation programs. Each scenario included the patient's age, diagnosis, severity of injury and information related to the patients' physical abilities, cognitive abilities, functional status, living environment and pre-morbid functioning. For each scenario, participants were asked to identify factors they would consider when assessing the patient's RP and to add any other pertinent factor(s) not included in the scenario. All identified factors related to patients' characteristics and will subsequently be referred to as 'patient-related factors'. In preparation of the group phase, each identified patient-related factor was written on a large card to be presented to the group in the next phase. 


\section{Group phase}

We initiated the group discussion by presenting participants a brief overview of their combined understanding of RP as well as the following definition which, to our knowledge, appears to be the most frequently cited in the literature: "an estimate of the individual's capability of co-operating with a rehabilitation program and making measured functional gains"' (Rentz, 1991). Although the term RP is widely used in clinical practice, we were unable to find a commonly accepted definition of this concept in the literature. We therefore asked the group to agree on a working definition of RP as a way to ensure that participants had a common understanding of the term before engaging in further discussions. Participants agreed on the following working definition, a slightly modified version of Rentz's definition: “estimate which takes into account a set of factors that enable a person with a disability following an $\mathrm{ABI}$ to participate in a rehabilitation program and to demonstrate measurable functional gains during the rehabilitation episode of care".

We then proceeded to the group phase of the TRIAGE methodology during which the cards containing factors identified by the participants in the individual phase were posted on the wall. The following question guided group discussions: "Which factors are essential to consider when occupational therapists assess the RP of patients with ABI?". The group had to decide whether to retain the factor as formulated (the factor was moved to a 'selection' section on the wall), eliminate the factor (the factor was moved to the 'wastebasket') or combine the factor with another one under an overarching factor (e.g. memory was moved to a 'grouping' section and later combined with other factors under the overarching factor named cognitive abilities). 
The entire group discussion lasted $2 \frac{1}{2} 2$ hours and was audio recorded; the verbatim was transcribed and imported into QDA miner software (Provalis Research) for data management.

\section{Analysis}

Participants' socio-demographic and clinical practice characteristics were analysed using descriptive statistics.

Data analysis was not required to address the first objective since the TRIAGE consensus technique produced a list of patient-related factors.

To address the second objective, we analysed the transcription of the group discussion in accordance with an interpretive description approach. The first author (PL) and a second coder (KD - an acute care occupational therapist with research experience using qualitative methodology and who was not a study participant) read the entire transcript to gain a general sense of the content's meaning. Then, they each independently analysed the transcript content by first generating initial codes for all meaningful ideas emerging from the data. When coding the data, a preliminary analytic framework was used when naming and categorising themes. Interpretive description approach acknowledges that the researcher will use theoretical knowledge and clinical background as a starting point to generate knowledge pertinent to clinical practice. In this study, Foster and Tilse's conceptual model of post-acute referral after traumatic brain injury was used as a preliminary analytic framework. Briefly, this model views clinicians as 'gatekeepers' of resources and their interpretive activities as central to post-acute care referral decisions. Clinicians' engage in processes of interpreting patients' characteristics and these 
interpretations are shaped by the immediate clinical context as well as the broader health care context (Foster \& Tilse, 2003). Since the TRIAGE technique already provided a list of patient-related factors deemed pertinent to the assessment of RP, this preliminary analytic framework was rather used to explore the influence of organisational factors and clinicians' interpretive activities on occupational therapists' perception of RP. Codes that emerged from the data but could not be categorised using this framework were further discussed amongst coders to explore their meanings and to examine how they related to other codes. This allowed for an emerging and broader conceptualisation of factors that influence occupational therapists' perception of RP. PL and KD developed a coding grid throughout the analytic process. A third coder (OM - acute care occupational therapist with research experience and who was not a study participant) was then asked to perform a final round of analysis using the coding grid while also letting other themes that did not relate to existing codes emerge from the data. PL and OM met for a final review of codes. PL wrote analytic memos after the focus group and during the data analysis as a way to rapidly capture thoughts occurring throughout data collection and analysis. These memos were used during analysis to aid in making sense of the data collected (Thorne, 2008).

\section{RESULTS}

Twelve occupational therapists ( 6 from acute care and 6 from inpatient rehabilitation) were recruited from facilities in Montreal, Canada. At the time of the study, eleven participants worked or had worked with persons with a stroke and four worked with persons with a traumatic brain injury. As a group, they had practiced an average of 13.5 years (ranging from 5.5 to 32 years of experience), and specifically with persons with ABI for a mean of 
11.7 years (ranging from 4 to 23 years). All acute care participants reported assessing RP: two assessed RP in $90 \%$ of their patients and four assessed RP in 100\% of their patients. The amount of time available to acute care occupational therapists to conduct these evaluations varied between 60 and 180 minutes. Inpatient rehabilitation participants estimated that, in general, between 5 and $20 \%$ of patients transferred to their hospital present poor RP.

\section{Patient-related factors}

In the individual phase, participants identified 51 patient-related factors influencing their perception of RP (Table 1). The group achieved consensus on 11 factors they perceived as essential to consider: medical status, pre-injury and post-injury functional status, cognitive abilities, behaviour, age, physical abilities, endurance, observed improvement post-injury, home environment, patient/family expectations. Table 2 presents factors identified in the individual phase and their grouping under these 11 overarching factors during the group phase. Participants specified that patient's medical status is assessed by the medical team and not by occupational therapists. However, information provided by the medical team or observations regarding some aspects of medical status made by occupational therapists are indispensable when determining a patient's RP (e.g. injury severity).

The analysis of the group discussion transcripts further revealed additional factors influencing participants' perception of RP. Two factors were consistent with Foster and Tilse's model: organisational factors and clinicians' interpretive activities (Figure 1). Four 
additional themes emerged directly from the group discussion: professional expertise, experiential knowledge, knowledge of scientific evidence, and concern for ethical decisions (Figure 1). Themes and subthemes are summarised in Table 3 and direct quotes are used in the following text to illustrate the themes as described in participants' own words.

\section{Organisational context}

All acute care participants reported having limited time available for assessing RP: '...sometimes it happens that we have only 60 minutes to assess a patient (...)' [acute careP10]. This creates constant pressure for rapid decision-making based on limited information obtained during a single short encounter with the patient. Participants also mentioned that the acute care context imposed limitations on their professional activities: '... in the [acute care] hospital, we do not have the time to provide intensive rehabilitation. (...) in acute care, [the patients] do not do much. They are washed, they are dressed, and they sit. We haven't really tested their abilities...' [acute care-P12]. The acute care context was described as an environment where rehabilitation is not the primary focus, with physicians insisting on discharging patients as soon as they are medically stable, and where nursing activities are focused on medical issues (e.g. washing and dressing the patient to be ready for medical tests or interventions) and not on rehabilitation. However, acute care participants felt that the multidisciplinary process in the acute care context facilitates the assessment process by providing a more comprehensive clinical picture of patients' RP:

'... in team meetings, we start from the patient, with the medical notes from the day the patient arrived at the hospital, this gives us an idea. I see the 
patient the same day, the physiotherapist sees the patient the next day. I talk with the physiotherapist who notes my results, I note the recovery of motor function, the team follows the patient. For me, assessing a patient's progress is a team activity, even if we each don't have much time [to assess]' [acute care-P10].

Participants working in both acute care and inpatient rehabilitation also reported that many aspects of the broader health care context influence the referral process to inpatient rehabilitation. Being cognisant of the services available in the continuum of care for $\mathrm{ABI}$ patients (or gaps in services) influences which patients are referred to inpatient rehabilitation. For example, a participant reported some patients are referred to inpatient rehabilitation although they may have benefited from outpatient rehab: '... since there is nothing else available, [some patients] are treated in inpatient rehabilitation by a whole [multidisciplinary] team even though it is only the occupational therapist that works with them one hour per day' [rehab-P3]. Participants also stressed that being aware of the rehabilitation admission criteria influences decisions related to RP and patient selection for inpatient rehabilitation. Some criteria are considered official while others are unofficial and based on clinical knowledge of the health care context: '...sometimes, we will say "no, he is still in restraints, he cannot, he cannot go [to rehab]', that we will hear often' [rehabP6]. Participants further mentioned that admission to rehabilitation is perceived as limited to those patients with the greatest potential because of the limited number of beds available: 'the problem is that there are lots of patients who would benefit from rehabilitation. However, because of the limited beds in rehabilitation (...) we have a good idea of the 
barriers for [admission to] rehabilitation' [rehab-P11]. Participants felt they sometimes have to advocate for patients who present with lower levels of RP:

if the patient presents with severe memory issues only [limiting learning ability], me, I will still often advocate for this, so that the patient can go to rehabilitation since the patient has never been taught compensatory strategies. But it often happens that they will not be admitted' [acute care$P 1]$.

Participants recognised long wait times for access to rehabilitation influenced how they select patients for rehabilitation: 'if the delays are one week, two weeks or three weeks, we understand that if we sent everyone to rehabilitation, the health care system would not be able to support that' [acute care-P12]. Finally, participants also mentioned that the capacity of care providers in rehabilitation to provide specific types of services (e.g. tracheostomy care, constant supervision) determines who will be considered a rehabilitation candidate: 'if the patient needs to be supervised at all times, then it happens that nursing [in inpatient rehabilitation] will say that they cannot provide supervision' [rehab-P11]. In sum, participants reported many organisational aspects of acute care, inpatient rehabilitation and the broader health care system, which influence how RP is perceived and how clinicians determine rehabilitation candidacy.

\section{Professional expertise}

Participants discussed the contribution of occupational therapy to the multidisciplinary assessment of RP by mentioning aspects of assessment congruent with their professional field of practice: 'I think when you have a patient who has problems with memory, and 
physically everything is okay, as occupational therapists we're usually the ones advocating for them, even just to have a chance at rehab' [acute care-P4]. 'Us, we evaluate the impact on the functional level' [rehab-P6]. Although occupational therapists consider many patient-related factors in their assessment of RP, some of these factors are also assessed by other team members according to their own professional field of practice: '...it's not up to us to decide if the patient is [medically] stable. It is the doctor who will judge that' [acute care-P12]. Therefore, recognising the strengths and limits of occupational therapy expertise helped participants to identify the factors upon which to focus their evaluation of RP (e.g. occupational therapists do not evaluate medical status but focus their assessment on cognition and functional status).

\section{Experiential knowledge}

Participants often referred specifically to their own clinical experience working with ABI patients when discussing which patient-related factors were the most important to consider. For example, when discussing how medical diagnosis impacts the evaluation of RP, a participant mentioned 'by experience, I know that haemorrhagic strokes, I will give them more time [before concluding on the patient's RP]' [acute care-PI]. Another participant said: 'the probability [of recovering function], that is what we think about with our experience' [rehab-P11]. Many similar references to experiential knowledge were heard throughout the group discussion and shaped their perception of RP. 


\section{Knowledge of scientific evidence}

Participants referred to scientific evidence on only two occasions. When discussing the impact of hemi neglect on RP, a participant said 'lots of studies have been conducted specifically on hemi neglect and prognosis in rehabilitation (...) it affects the outcome of rehabilitation' [rehab-P6]. When discussing the impact of age on the RP assessment, participants referred to clinical practice guidelines: '[impact of age on rehabilitation outcome] is based on clinical practice guidelines, on the Canadian recommendations, I think' [acute care-P10].

\section{Ethical considerations}

Although we did not perform an extensive in-depth analysis of ethical dilemmas, participants did voice concerns for making the right decisions regarding patients' RP. These concerns were congruent with the four basic ethical principles of autonomy, beneficence, nonmaleficence and justice (Beauchamp \& Childress, 2001). In regards to autonomy, participants reported it is rare to see patients in acute care set their own objectives' [acute care-P1]. Therefore clinicians had to listen to patient and family expectations to attempt to identify therapeutic goals to be pursued in rehabilitation that would align with patients' wishes. The principles of beneficence/nonmaleficence refer to doing good and avoiding actions that can cause harm. The group occasionally conveyed aspects of this principle. For example, when discussing the challenge of predicting which patients may improve with rehabilitation, a participant said: 'is it our prognosis [in acute care] that is correct? Maybe not, maybe we think that this patient will not recover but the patient may have been able to' [acute care-P12]. There were also many references to the 
principle of justice. For example, when discussing the challenge in admitting patients with only specific cognitive difficulties to inpatient rehabilitation, a participant said 'patients who present with only memory problems, that happens often, I will fight for these patients for a chance at rehabilitation' [acute care-P1]. Overall, participants' concerns for ethical decisions revolved around balancing between providing rehabilitation to all who need rehabilitation, while at the same time not wasting limited rehabilitation resources.

\section{Interpretative activities}

The analysis of the group discussion further revealed that clinicians interpret patient characteristics in the light of the other factors and the interpretive process shapes the assessment of RP. Three interpretive activities were found to underlie the estimation of patients' RP: predicting improvement, identifying appropriate therapeutic goals for rehabilitation, and judging which patients meet inpatient rehabilitation admission criteria (Figure 1). Clinicians attempted to predict if their patients' will improve by considering various patient characteristics and linking these characteristics to possible outcomes based on their own past clinical experience or on scientific evidence: 'it is how the client progresses in acute care, if we see some gains in terms of independence, we think 'well, he will continue to improve' [acute care-P1]. Participants also mentioned attempting to determine appropriate rehabilitation goals, goals that would seem appropriate to pursue in the context of inpatient rehabilitation and that would also be aligned with patient and family expectations. This type of interpretive activity requires clinicians be knowledgeable not only of their patients' needs and expectations but also of the type of post-acute care rehabilitation services available and what specific interventions are offered in inpatient 
rehabilitation to address patients' needs. Participants mentioned that inpatient rehabilitation is focused on basic ADLs ('[in inpatient rehabilitation] we aim to improve independence for ADLs' [acute care-P12]) but pursuing goals related to quality of life was also appropriate for inpatient rehabilitation ('patients do benefit from inpatient rehabilitation if they have the potential to improve their quality of life or lessen the burden of care [rehab-P7].). Furthermore, to estimate a patient's RP, clinicians also have to judge if the patient's status or level of functioning meets admission criteria established by inpatient rehabilitation institutions. Participants referred to official admission criteria that reflect minimal pre-requisites to receive rehabilitation interventions: 'it is a prerequisite [for admission to inpatient rehabilitation], if the patient is not medically stable, you don't even question' [acute care-P10].

\section{DISCUSSION}

To our knowledge, this is the first study to examine factors influencing occupational therapists' perceptions of patients' RP after ABI. Our results identified eleven patient characteristics considered as essential to the assessment of RP: age, medical status, preinjury and post-injury functional status, cognitive and physical abilities, behaviour, home environment, endurance, observed improvement post-injury and patient/family expectations. Factors other than patient-related ones were also found to influence occupational therapists' perceptions of a patient's RP, i.e. organisational contexts, occupational therapists' professional expertise, experiential knowledge, knowledge of scientific evidence, and ethical concerns. Moreover, we found that therapists interpret patient-related factors while considering all of the other non-patient related factors in an 
attempt to predict recovery, identify appropriate therapeutic goals, and judge which patient is a rehabilitation candidate.

Amongst the eleven patient-related factors deemed essential to consider by focus group participants, age, functional status and cognitive abilities measured in the acute phase after ABI have also been found to be predictive of functional outcome following rehabilitation (Fleming, Tooth, Hassell, \& Chan, 1999; Hakkennes, Brock, \& Hill, 2011). Hence, considering the important role of occupational therapists in the evaluation of functional status, it is becoming clearer that occupational therapists can contribute meaningfully to multidisciplinary teams' predictions regarding patients most likely to recover. Our findings are also congruent with recent studies that have examined factors influencing physicians' decisions to admit patients to inpatient rehabilitation. Here age, pre-morbid function, physical function, behaviour, cognitive function, social support, functional gains during acute hospitalisation and ability to participate to therapy during acute hospitalisation have been identified as influential factors in physicians' decisions (Hayward et al., 2014; Kennedy et al., 2012). A recent study examining health care professionals' perspectives further identified that patient and family expectations also influence professionals' perceptions of patients' RP (Burton, Horne, Woodward-Nutt, Bowen, \& Tyrrell, 2015). It therefore seems that patient-related factors identified in this study provide a relatively comprehensive picture of factors deemed essential to consider when assessing RP. Further studies are required to better understand how occupational therapists objectively assess these factors in acute care and how they incorporate these results in their clinical reasoning underlying their assessment of RP. 
Our results also suggest that clinicians' assessment of RP goes beyond only assessing patient characteristics. We found additional factors influencing occupational therapists' perception of patients' RP, i.e. organisational factors, occupational therapists' professional expertise, experiential knowledge, knowledge of scientific evidence and ethical concerns. These factors are consistent with factors found to influence discharge decision-making (Jette, Grover, \& Keck, 2003) and clinical reasoning (Carrier, Levasseur, Bedard, \& Desrosiers, 2010). Therefore, assessing RP may be far more complex then solely considering patients' characteristics such as suggested in decision-making algorithms or admission criteria. Our findings also provide insight into the interpretive activities that may be involved in the clinical reasoning process underlying the assessment of RP. Occupational therapists talked about engaging in active interpretation of patients' characteristics in order to predict recovery, to identify appropriate therapeutic goals and to judge which patient is a rehabilitation candidate. Hence, future studies examining clinical reasoning underlying the assessment of RP in actual acute care settings are required to better understand how clinicians interpret and determine RP. Furthermore, recent models of evidence-based practice have advocated that patient characteristics, professional expertise and scientific evidence are essential sources of information that influence clinical decisions, while environmental contexts and ethical considerations also shape these decisions (Chabon, Morris, \& Lemoncello, 2011; Satterfield et al., 2009). Clinical reasoning that incorporates elements of evidence-based practice is the cornerstone of professional competency and results of our study suggest that as a group, experienced occupational therapists consider several elements of evidence-based practice when discussing the assessment of RP. For students or novice clinicians to acquire competence 
in RP assessment, educators or mentors should consequently focus not only on patient characteristics but also address all other aspects of evidence-based clinical reasoning (scientific evidence, professional expertise, environmental contexts and ethical considerations).

\section{Limitations}

Although appropriate for an exploratory study, a small sample size and a single focus group with occupational therapists selected from a more or less homogeneous model of service delivery of universal access likely influenced the data collected. Clinicians need to reflect on how the findings of this study apply to their clinical practice within their own organisational environment. Also, we used the term RP because it is often used in clinical practice but there is no clear definition or widespread agreement on this concept in the literature. Although this study did not aim to define the concept of RP, we had to make sure participants had a common understanding by asking them to agree on a working definition of RP prior to the focus group. Readers should therefore judge if this working definition and the subsequent results apply to their clinical practice. Furthermore, using a consensus seeking methodology, we obtained patient-related factors identified by participants as essential to consider; participants were not asked to provide a definition of each factor since this was not an aim of the study. Although a definition for each factor is not provided, readers can refer to Table 2 to get a sense of the more specific factors that were grouped under the overarching patient-related factors identified by group consensus. This may provide readers with a better sense of what was meant by the 11patient-related factors for which group consensus was obtained. Lastly, the results are based on verbal 
reports and not observed behaviours in actual clinical context. Future studies are required to validate how findings from this study unfold in actual clinical practice.

\section{CONCLUSIONS}

Access to inpatient rehabilitation services is crucial for optimising recovery for individuals with $\mathrm{ABI}$ but determining who should have access to rehabilitation can be challenging in current health care practices. The results of this exploratory study suggest that assessing RP is a complex process that goes beyond strictly appraising patients' characteristics and purely forecasting patient's future functioning. Occupational therapists interpret patient characteristics in light of additional factors related to their own professional expertise, experiential knowledge, knowledge of scientific evidence, organisational context and ethical considerations. Clinicians should pay more attention to these factors when making evidence-based decisions regarding patients' potential to benefit from rehabilitation. The complexity of decision-making regarding the assessment of RP calls for more in-depth research on the underlying clinical reasoning used by rehabilitation professionals when selecting appropriate patients for rehabilitation.

\section{Acknowledgements}

The authors would like to thank the participants involved in this study and their institutions as well as the research assistants who participated in data analysis. This study received financial support from the "Ordre des ergothérapeutes du Québec" and the Quebec Rehabilitation Research Network. 


\section{REFERENCES}

ABIKUS Guideline Development Group. (2007). ABIKUS Evidence Based

Recommendations for Rehabilitation of Moderate to Severe Acquired Brain Injury (2007) Retrieved June 29, 2015, from

http://www.abiebr.com/pdf/abikus_aug_07.pdf

Beauchamp, T., \& Childress, J. (2001). Principles of biomedical ethics (5th ed.). Oxford: Oxford University Press.

Britton, L., Rosenwax, L., \& McNamara, B. (2015). Occupational therapy practice in acute physical hospital settings: Evidence from a scoping review. Aust Occup Ther J, 62(6), 370-377. doi: 10.1111/1440-1630.12227

Burton, C. R., Horne, M., Woodward-Nutt, K., Bowen, A., \& Tyrrell, P. (2015). What is rehabilitation potential? Development of a theoretical model through the accounts of healthcare professionals working in stroke rehabilitation services. Disability and Rehabilitation, 37(21), 1955-1960. doi: 10.3109/09638288.2014.991454

Carrier, A., Levasseur, M., Bedard, D., \& Desrosiers, J. (2010). Community occupational therapists' clinical reasoning: identifying tacit knowledge. Australian Occupational Therapy Journal, 57(6), 356-365. doi: 10.1111/j.14401630.2010.00875.x

Casaubon, L. K., Boulanger, J. M., Glasser, E., Blacquiere, D., Boucher, S., Brown, K., . .. Lindsay, P. (2016). Canadian Stroke Best Practice Recommendations: Acute Inpatient Stroke Care Guidelines, Update 2015. International Journal of Stroke, 11(2), 239-252. doi: 10.1177/1747493015622461 
Chabon, S., Morris, J., \& Lemoncello, R. (2011). Ethical deliberation: a foundation for evidence-based practice. Seminars in speech and language, 32(4), 298-308. doi: $10.1055 / \mathrm{s}-0031-1292755$

Conroy, B. E., DeJong, G., \& Horn, S. D. (2009). Hospital-based stroke rehabilitation in the United States. Top Stroke Rehabilitation, 16(1), 34-43. doi: 10.1310/tsr160134

Crennan, M., \& MacRae, A. (2010). Occupational therapy discharge assessment of elderly patients from acute care hospitals. Physical \& Occupational Therapy in Geriatrics, 28(1), 33-43. doi: 10.3109/02703180903381060

Cullen, N. (2007). Canadian healthcare perspective in traumatic brain injury rehabilitation. Journal of Head Trauma Rehabilitation, 22(4), 214-220. doi: 10.1097/01.HTR.0000281836.54602.6d

Cullen, N., Meyer, M.J., Aubut, J.-A., Bayley, M., \& Teasell, R. (2014). Efficacy and Models of Care Following an Acquired Brain Injury. Evidence-Based Review of Moderate to Severe Acquired Brain Injury. Retrieved June 29, 2015, from http://www.abiebr.com

Fleming, J., Tooth, L., Hassell, M., \& Chan, W. (1999). Prediction of community integration and vocational outcome 2-5 years after traumatic brain injury rehabilitation in Australia. Brain Injury, 13(6), 417-431. doi:

$10.1080 / 026990599121476$

Foster, M., \& Tilse, C. (2003). Referral to rehabilitation following traumatic brain injury: a model for understanding inequities in access. Social Science \& Medicine, 56(10), 2201-2210. 
Gervais, M., \& Pépin, G. (2002). TRIAGE: A new group technique gaining recognition in evaluation. Evaluation journal of Australasia, 2(2), 45-49.

Government of South Australia. (2012). Acquired brain injury model of rehabilitation for South Australia. Retrieved June 30, 2016, from https://http://www.sahealth.sa.gov.au

Hakkennes, S. J., Brock, K., \& Hill, K. D. (2011). Selection for inpatient rehabilitation after acute stroke: a systematic review of the literature. Archives of Physical Medicine and Rehabilitation, 92(12), 2057-2070. doi: 10.1016/j.apmr.2011.07.189

Hayward, K.S., Aitken, P.D., Barker, R.N., \& Brauer, S.G. (2014). Admission to and continuation of inpatient stroke rehabilitation in Queensland Australia: A survey of Factors that contribute to the Consultant's Decision. Brain impairment, 15(2), 88-98. doi: 10.1017/BrImp.2014.12

Ilett, P. A., Brock, K. A., Graven, C. J., \& Cotton, S. M. (2010). Selecting patients for rehabilitation after acute stroke: are there variations in practice? Archives of Physical Medicine and Rehabilitation, 91(5), 788-793. doi: 10.1016/j.apmr.2009.11.028

Jette, D. U., Grover, L., \& Keck, C. P. (2003). A qualitative study of clinical decision making in recommending discharge placement from the acute care setting. Physical Therapy, 83(3), 224-236.

Jourdan, C., Bayen, E., Bosserelle, V., Azerad, S., Genet, F., Fermanian, C., . . Azouvi, P. (2013). Referral to rehabilitation after severe traumatic brain injury: results 
from the PariS-TBI Study. Neurorehabilitation and Neural Repair, 27(1), 35-44. doi: $10.1177 / 1545968312440744$

Kennedy, G. M., Brock, K. A., Lunt, A. W., \& Black, S. J. (2012). Factors influencing selection for rehabilitation after stroke: a questionnaire using case scenarios to investigate physician perspectives and level of agreement. Arch Phys Med Rehabil, 93(8), 1457-1459. doi: 10.1016/j.apmr.2011.11.036

Lamontagne, M. E., Routhier, F., \& Auger, C. (2013). Team consensus concerning important outcomes for augmentative and alternative communication assistive technologies: a pilot study. Augmentative and Alternative Communication, 29(2), 182-189. doi: 10.3109/07434618.2013.784927

Lindsay, M.P., Gubitz, G., Bayley, M., Hill, M.D. , Davies-Schinkel, C., Singh, S., \& Phillips, S. . (2010). Canadian Best Practice Recommendations for Stroke Care (update 2010). Retrieved June 29, 2015, from http://www.strokebestpractices.ca/wpcontent/uploads/2011/04/2010BPR_ENG.pdf

National Stroke Foundation. (2015). Acute stroke services framework 2015. Retrieved June 30, 2016, from https://strokefoundation.com.au/ /media/strokewebsite/resources/treatment/nsf11 59_acuteframework2015.ashx?la=en

Pereira, S., Graham, J. R., Shahabaz, A., Salter, K., Foley, N., Meyer, M., \& Teasell, R. (2012). Rehabilitation of individuals with severe stroke: synthesis of best evidence and challenges in implementation. Top Stroke Rehabililitation, 19(2), 122-131. doi: 10.1310/tsr1902-122 
Provalis Research. QDA miner (Version 4.0). Montréal, Québec, Canada. Retrieved from http://provalisresearch.com/products/qualitative-data-analysis-software/

Putnam, K., De Wit, L., Schupp, W., Beyens, H., Dejaeger, E., De Weerdt, W., . . Leys, M. (2007). Inpatient stroke rehabilitation: a comparative study of admission criteria to stroke rehabilitation units in for european centres. Journal of rehabilitation medicine, 39, 21-26. doi: 10.2340/16501977-0006

Rentz, D.M. . (1991). The assessment of rehabilitation potential: cognitive factors In Hartke RJ ed. (Ed.), Psychological aspects of geriatric rehabilitation. Gaithersburg, MD: Aspen.

Satterfield, J. M., Spring, B., Brownson, R. C., Mullen, E. J., Newhouse, R. P., Walker, B. B., \& Whitlock, E. P. (2009). Toward a transdisciplinary model of evidencebased practice. Milbank Quartely, 87(2), 368-390. doi: 10.1111/j.14680009.2009.00561.x

Schwamm, L. H., Pancioli, A., Acker, J. E., 3rd, Goldstein, L. B., Zorowitz, R. D., Shephard, T. J., . . Adams, R. J. (2005). Recommendations for the establishment of stroke systems of care: recommendations from the American Stroke Association's Task Force on the Development of Stroke Systems. Circulation, 111(8), 1078-1091. doi: 10.1161/01.CIR.0000154252.62394.1E

Swaine, B., Cullen, N., Bayley, M., Lavoie, A., Sirois, M.J., Turgeon, A., \& Messier, F. (2011). Who gets admitted to rehabilitation? Perceptions of clinicians and managers in two Canadiann provinces working in clinical programs for persons with a brain injury. Annals of Physical and Rehabilitation Medicine, 54s, e278. doi: 10.1016/j.rehab.2011.07.214 
Swaine, B., Dutil, E., Demers, L., \& Gervais, M. (2003). Evaluating clients' perceptions of the quality of head injury rehabilitation services: development and validation of a questionnaire. Brain Inuryj, 17(7), 575-587. doi:

$10.1080 / 0269905031000088568$

Thorne, S. (2008). Interpretive description. Walnut Creek: California: Left Coast Press inc.

Veillette, N., Demers, L., Dutil, E., \& McCusker, J. (2009). Development of a functional status assessment of seniors visiting emergency department. Archives of Gerontology and Geriatrics, 48(2), 205-212. doi: 10.1016/j.archger.2008.01.009 World Health Organization. (2003). The World health report : 2003 : shaping the future. Retrieved June 29, 2015, from http://www.who.int/whr/2003/en/ 
TABLE 1: Individual phase results

\section{1 factors influencing occupational therapists' perception of acquired brain injury patients' rehabilitation potential}

Ability to follow simple instructions

Ability to formulate goals

Actual level of autonomy

Age

Apraxia

Attention

Behaviour

Client and family expectations

Cognitive abilities

Collaboration

Coma

Communication abilities

Compliance to interventions

Culture

Diagnosis

Discharge disposition following rehab

Emotional status

Executive functions
Endurance

Expected ability to resume social roles

Expected improvement for usual activities

Financial resources

Functional level

Frustration tolerance

Impulsivity

Judgement

Learning ability

Length of acute care hospitalization

Length of coma state

Medical prognosis

Medical status

Memory

Mobility

Motivation

Motor abilities

Observed improvement in autonomy in acute care
Orientation (time, place, person)

Participation in self-care activities

Perceptual abilities

Physical environment

Post traumatic amnesia

Pre-morbid status

Psychological status

Risk of falls

Self-awareness

Sensory abilities

Side effect of medical treatments

Social environment

Swallowing abilities

TBI severity

Time post-injury 
TABLE 2: Group phase results

\section{Grouping of factors under 11 overarching patient-} related factors after group consensus*

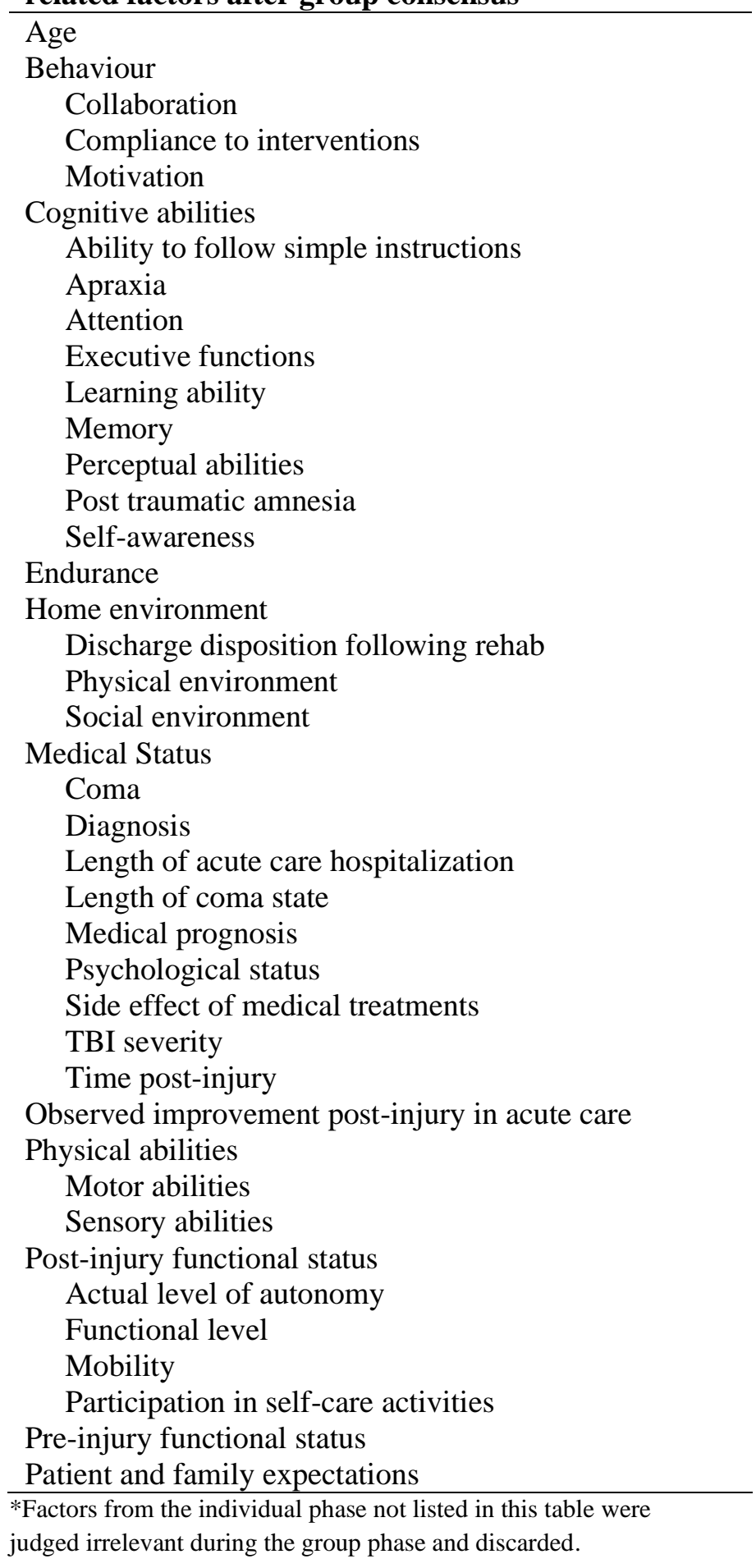


TABLE 3: Factors other than patient-related factors influencing occupational therapists' perception of rehabilitation potential

\begin{tabular}{|c|c|}
\hline \multirow{3}{*}{$\begin{array}{l}\text { Themes } \\
\text { Organizational context* }\end{array}$} & Subthemes \\
\hline & $\begin{aligned} & \text { Acute care environment } \\
& \text { - } \text { Limited time for assessment } \\
& \text { - } \text { Focus on medical activities limits activities of } \\
& \text { rehabilitation professionals } \\
& \text { - } \text { Multidisciplinary approach facilitates } \\
& \text { comprehensive assessment }\end{aligned}$ \\
\hline & $\begin{aligned} & \text { Broader health care environment } \\
& \text { - } \text { Availability of post-acute rehabilitation services } \\
& \text { - } \text { Inpatient rehabilitation admission criteria } \\
& \text { - } \text { Bed shortages in inpatient rehabilitation } \\
& \text { - } \text { Long wait times to access inpatient } \\
& \text { rehabilitation } \\
& \text { - } \text { Human workforce capacity in inpatient } \\
& \text { rehabilitation }\end{aligned}$ \\
\hline Professional expertise & $\begin{array}{l}\text { OT's field of practice } \\
\text { Respect for other team members' field of expertise }\end{array}$ \\
\hline Experiential knowledge & Clinical experience with ABI patients \\
\hline & $\begin{array}{l}\text { Research studies } \\
\text { Clinical practice guidelines }\end{array}$ \\
\hline Ethical considerations & $\begin{array}{l}\text { Justice } \\
\text { Beneficence/nonmaleficence } \\
\text { Autonomy }\end{array}$ \\
\hline Interpretative activities* & $\begin{array}{l}\text { Predicting improvement } \\
\text { Identifying appropriate therapeutic goals for } \\
\text { rehabilitation } \\
\text { Judging which patients meet inpatient rehabilitation } \\
\text { admission criteria }\end{array}$ \\
\hline
\end{tabular}

* Themes derived from Foster and Tilse's conceptual model for understanding post-acute care referral (2003) 


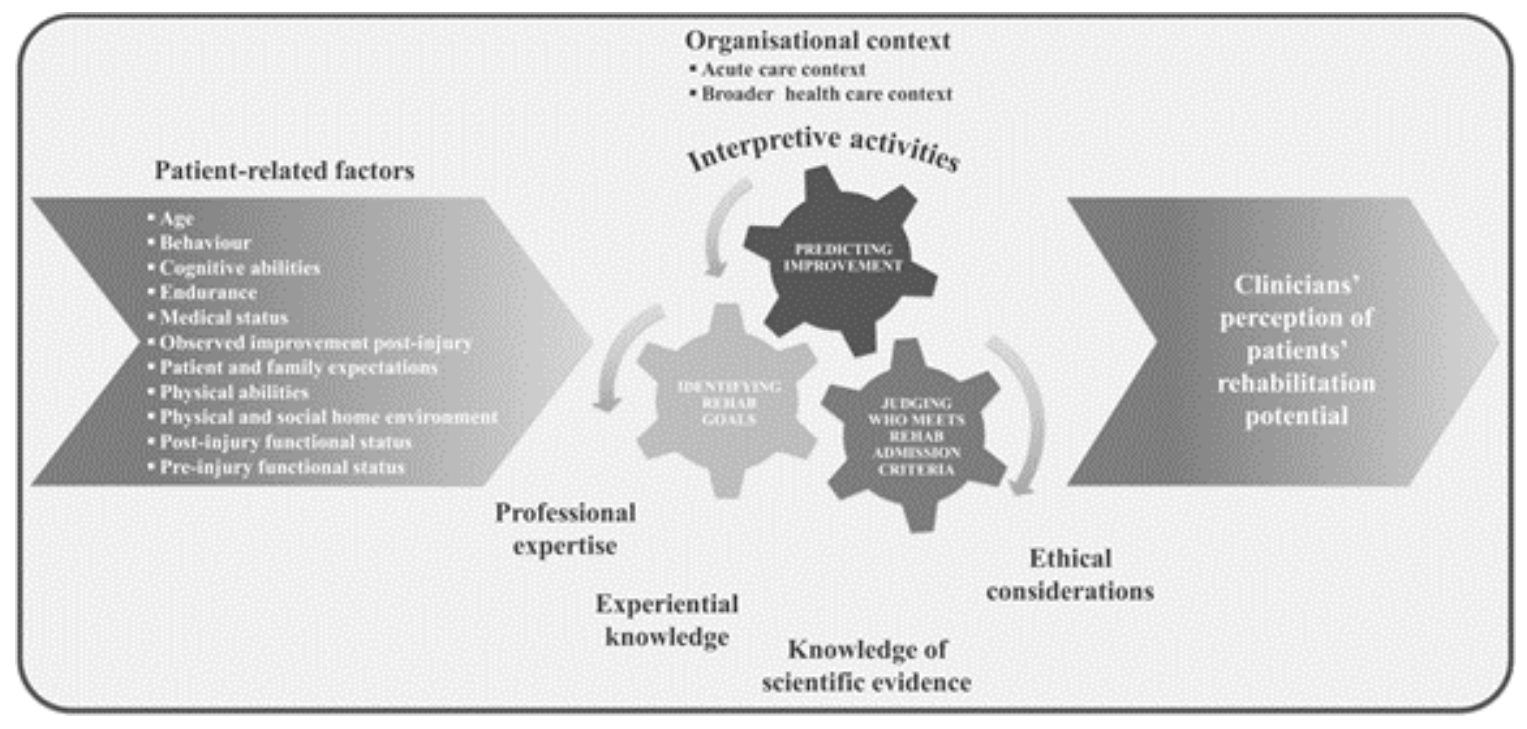

FIGURE 1: Factors influencing occupational therapists' perception of ABI patients' RP for inpatient rehabilitation. 